\title{
Why Clothes Don't Fall Apart: Tension Transmission in Staple Yarns
}

\author{
Patrick B. Warren, ${ }^{1, *}$ Robin C. Ball, ${ }^{2, \dagger}$ and Raymond E. Goldstein ${ }^{3, \ddagger}$ \\ ${ }^{1}$ Unilever R\&D Port Sunlight, Quarry Road East, Bebington, Wirral CH63 3JW, United Kingdom \\ ${ }^{2}$ Department of Physics, University of Warwick, Coventry CV4 7AL, United Kingdom \\ ${ }^{3}$ Department of Applied Mathematics and Theoretical Physics, University of Cambridge, \\ Wilberforce Road, Cambridge CB3 OWA, United Kingdom
}

(Received 22 March 2017; revised manuscript received 9 February 2018; published 13 April 2018)

\begin{abstract}
The problem of how staple yarns transmit tension is addressed within abstract models in which the Amontons-Coulomb friction laws yield a linear programing (LP) problem for the tensions in the fiber elements. We find there is a percolation transition such that above the percolation threshold the transmitted tension is in principle unbounded. We determine that the mean slack in the LP constraints is a suitable order parameter to characterize this supercritical state. We argue the mechanism is generic, and in practical terms, it corresponds to a switch from a ductile to a brittle failure mode accompanied by a significant increase in mechanical strength.
\end{abstract}

DOI: 10.1103/PhysRevLett.120.158001

In his celebrated Dialogues Concerning Two New Sciences, Galileo identified a fascinating puzzle in the mechanics of ropes [1]. His fictitious discussant Salviati asks: "How are fibers, each not more than two or three cubits in length, so tightly bound together in the case of a rope one hundred cubits long that great force is required to break it?" Galileo's answer to this is to assert that "the very act of twisting causes the threads to bind one another in such a way that when the rope is stretched...the fibers break rather than separate from each other." From a modern perspective, we would say that the mechanical integrity of ropes derives from frictional contacts between fibers, and Galileo's rope problem is but one exemplar of a host of related frictional phenomena in fiber assemblies, of which perhaps the canonical case is the "staple" yarn [2-4]. Spun from fibers only $2-3 \mathrm{~cm}$ long [5], such a yarn is nevertheless patently capable of transmitting tension over indefinite distances. Accompanying these seemingly innocuous puzzles is an even more existential question: why don't clothes fall apart? After all, like Galileo's rope and the staple yarn, woven fabrics and sewn garments are only held together by friction.

A typical yarn (Fig. 1) is 100 fibers in cross section, and there are likely several frictional contacts per pitch length $(\sim 100 \mu \mathrm{m})$, per fiber; hence, we estimate $\gtrsim 50$ contacts per fiber and an overall contact density $10^{3}-10^{4} \mathrm{~cm}^{-1}$. Clearly, the problem of tension transmission in such a structure is a

Published by the American Physical Society under the terms of the Creative Commons Attribution 4.0 International license. Further distribution of this work must maintain attribution to the author(s) and the published article's title, journal citation, and DOI. problem in statistical physics. Here, we introduce and explore a class of abstract yarn models that isolate the key frictional ingredients of such a problem. Our analysis supports the idea that given sufficient friction and contact points, a random fiber assembly can in principle transmit an indefinitely large tension, by means of a collective friction locking mechanism that resembles a percolation transition.

The underlying premise is that normal forces acting between pairs of fibers facilitate tension transfer between fibers. The Amontons-Coulomb friction laws [6] then imply there is an upper bound on the tension $\Delta T$ that can be transferred before slip occurs. Away from the fiber ends, the fibers are in a tension-dominated regime even under models loads [7]; hence, the tension transfer "cap" can be expressed as $|\Delta T| \leq \lambda T_{m}$, where $T_{m}$ is the mean tension in the notionally overwrapped fiber [8] and $\lambda$ is

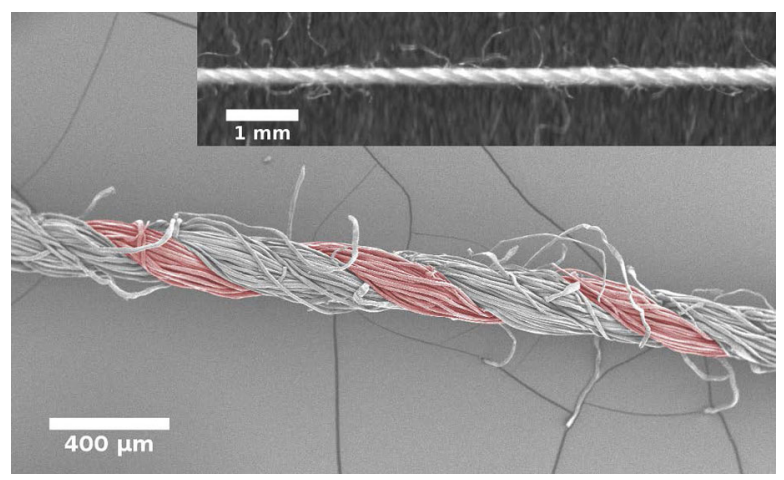

FIG. 1. A Gütermann cotton sewing thread. The composite 3-ply structure prevents untwisting under load. One ply (yarn) has been artificially tinted to emphasize the structure. Note the halo of stray fiber ends. Main image: SEM (Hitachi S-3400N); inset: flatbed scanner (Canon LiDE 220). 
what we term a tension transfer coefficient. In the spirit of the approach, we shall take the transfer coefficients from a random distribution to reflect the quenched disorder rather than attempting to solve the "inner" elastic problem [9] for each pair of fibers. The key insight is that if $\langle\lambda\rangle$ is large enough, this mechanism "bootstraps" a percolation transition for tension transmission.

We accommodate the remnant yet singular effect of bending stiffness in a lower bound to the tension in the fiber ends, which we estimate as $T^{*} \lesssim 1 \mathrm{mN}$ [10]. This is illustrated in Fig. 3(a), where the tension in each section of fiber between frictional contacts is shown building from zero at the free end. What $T^{*}$ means in practice is that we expect the percolation transition to correspond to a switch from a "ductile" failure mode where the yarn fails by fiber slippage, at around $T^{*}$ per fiber, cf. [11], to a "brittle" failure mode where the failure mechanism is fiber breakage, at $T^{\ddagger} \approx 20-130 \mathrm{mN}$ per fiber [12]. As we shall argue, twisting fibers together (in the manner of Galileo) pushes the assembly over the percolation threshold, resulting in perhaps a 100-fold increase in the tensile strength. Note that the scale separation between $T^{*}$ and $T^{*}$ means there is a significant loading regime, of practical relevance, where tension can only be carried by the percolation mechanism identified in the present work.

Given the transfer coefficients, the problem of computing the set of tensions $T_{i}$ in the fiber elements translates into a system of linear inequalities that can be solved by techniques imported from linear programing (LP). From this perspective, the question of whether the yarn transmits an arbitrarily large tension becomes a linear satisfiability problem. In this form it is fairly easy to show that $T^{*}$ is "irrelevant," in the language of renormalization group theory [13], and as such, we can carry out all our calculations setting $T^{*}=0$ [14]. Our approach shares elements with Bayman's "theory of hitches" [15], although in our model, a yarn is more akin to a random continuous splice comprised of many short fibers, rather than single-rope hitches.

To explain the above, we introduce a "toy" model of an actual splice, shown in Fig. 2. Suppose that the tensions in the various elements are as in Fig. 2(b), and the transfer coefficients are $\lambda_{1}$ and $\lambda_{2}$. Then,

$$
\begin{array}{ll}
\left|T_{1}-T_{0}\right| \leq \frac{1}{2} \lambda_{1}\left(T_{0}+T_{1}\right), & T_{0}=T_{1}+T_{2}, \\
\left|T_{2}-T_{3}\right| \leq \frac{1}{2} \lambda_{2}\left(T_{2}+T_{3}\right), & T_{1}+T_{2}=T_{3},
\end{array}
$$

where the inequalities are the tension transfer caps, and the equalities are force balance constraints. As mentioned, we simplify by assuming tension-free fiber ends, and in this particular, we case make a judicious choice for the overwrapping direction (otherwise, the splice would unravel). We define the LP objective function $z=\sum T_{i}$, and determine the percolation threshold by requiring $z>0$.
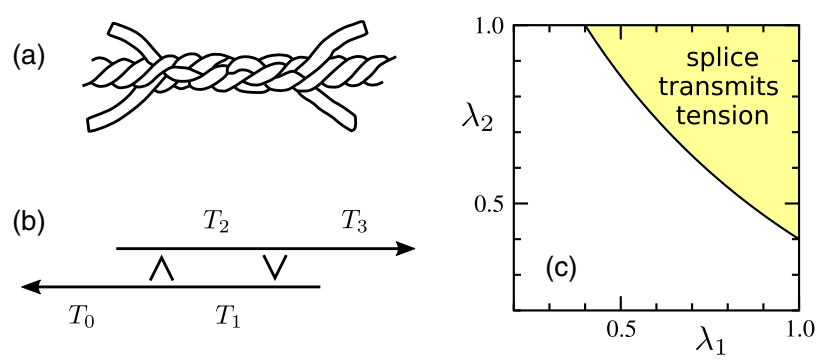

FIG. 2. (a) A "short" splice between two laid ropes [16]. (b) A schematic "toy" model of splice with labeled tensions (the $\vee$ and $\wedge$ shapes indicate the pinning direction). (c) A state space showing region (shaded) where tension can be transmitted.

This, together with Eqs. (1) and the constraints $T_{i} \geq 0$, specifies the LP problem.

This case can be solved by hand. Defining $x=T_{1} / T_{0}$ and $1-x=T_{2} / T_{0}$, with $0 \leq x \leq 1$, the caps yield $x \geq$ $\left(1-\frac{1}{2} \lambda_{1}\right) /\left(1+\frac{1}{2} \lambda_{1}\right)$ and $x \leq \lambda_{2} /\left(1+\frac{1}{2} \lambda_{2}\right)$. A solution thus requires $\left(1-\frac{1}{2} \lambda_{1}\right) /\left(1+\frac{1}{2} \lambda_{1}\right) \leq \lambda_{2} /\left(1+\frac{1}{2} \lambda_{2}\right)$, or $\lambda_{1}+\lambda_{2}+$ $\frac{3}{2} \lambda_{1} \lambda_{2} \geq 2$. If this inequality is satisfied, one is in a "locked" state where there are unbounded solutions with $z \rightarrow \infty$. Intuitively [Fig. 2(c)], such solutions exist in the highfriction region. Determining the value of $x$ (i.e., the individual tensions) in the supercritical locked state is complex; it may depend on the history of loading, forces beyond static friction, or the frictional contacts may adapt to the load, altering the transfer coefficients.

We now describe the abstract yarn model that captures the essential features of load transmission in fiber bundles by this mechanism. Shown in Fig. 3(a), it treats a yarn as a collection of randomly overlapped near-parallel fibers, each of finite length $N$, in units of the number of frictional contacts (pinning points). The structure comprises $m$ rows each of $r$ fibers, with a random longitudinal offset in each row, and it repeats periodically in the transverse and longitudinal directions [in Fig. 3(a), $m=6, N=8$, and $r=2]$. The pinning assignments in each column shown in Fig. 3(a) are randomly shuffled to mimic the random meandering of fibers through the structure. In the model, there are $(N+1) m r$ tensions $T_{i} \geq 0$ and $\frac{3}{2} N m r$ constraints coming from $\frac{1}{2} N m r$ pinning points. Thus, for $N>2$, the problem is potentially overconstrained, and solutions with $\sum T_{i}>0$ are possible only if there is sufficient "slack" in the tension transfer caps. As mentioned, the tension transfer coefficients $\lambda_{i}$ are independent and identically distributed random variables, with mean $\langle\lambda\rangle$ and distribution relative width $\sigma$. For each structure, we solve numerically for the onset of linear satisfiability as $\langle\lambda\rangle$ increases, then average over $10^{3}-10^{4}$ samples. Figure $3(\mathrm{~b})$ shows the dependence of the critical $N\langle\lambda\rangle$ on the fiber length $N$. In this representation, the results are insensitive to the model details, verifying our claim that for sufficiently long fibers there is a generic percolation transition in this model. Solving for the $T_{i}$, just above the threshold, yields insight into the 


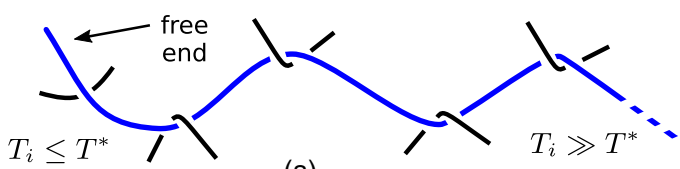

(a)
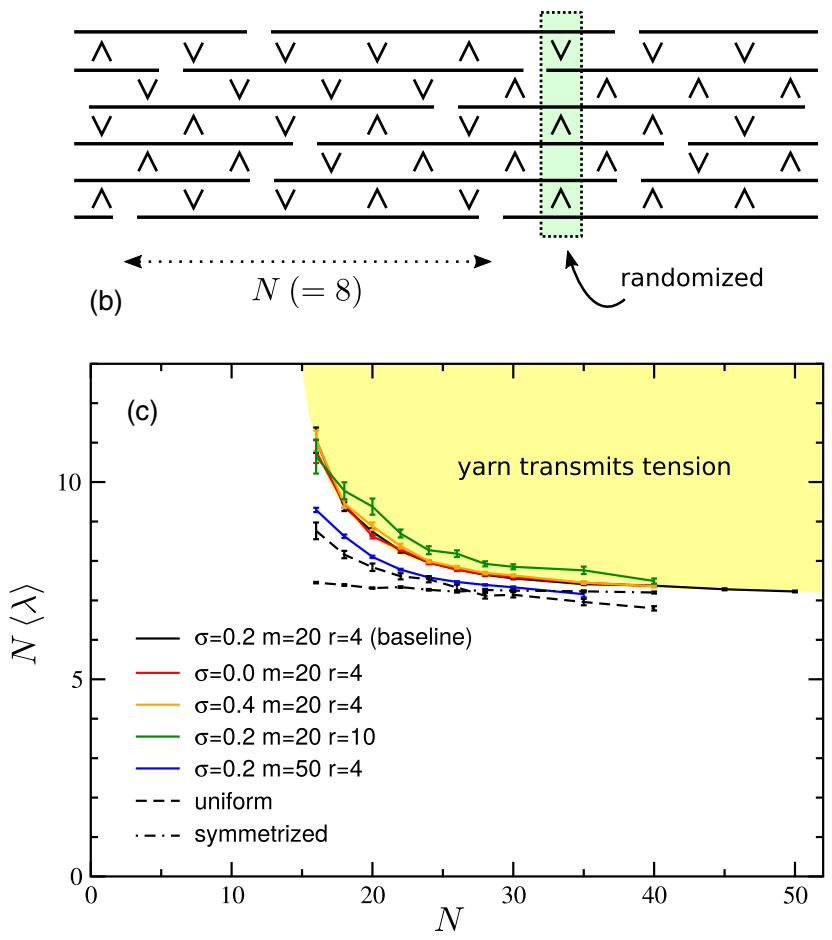

FIG. 3. (a) A fiber meandering through the yarn structure accumulates tension by means of frictional contacts (angles are exaggerated in this schematic). (b) An abstract yarn model in the notation of Fig. 2(b). For the baseline model the pinning assignments are randomly shuffled in each vertical column. (c) The critical value of $N\langle\lambda\rangle$ as a function of fiber length $N$, for different values of $m, r$, and the width $\sigma$ of the tension transfer coefficient distribution. We also considered a "uniform" version without random vertical shuffling (but with random pinning directions), and a "symmetrized" version in which $T_{m}$ is the mean tension in all fiber elements participating in a frictional contact.

percolating system of forces. Thus, Fig. 4 shows how tension in a fiber builds from zero at the free ends, attaining a maximum in the middle, and the inset shows the distribution of tensions in individual fiber elements.

For $N \gtrsim 30$, the percolation threshold is roughly constant at $N\langle\lambda\rangle \approx 7.3 \pm 0.2$. In the tension-dominated regime, and in the limit of a small turning angle $\theta$, the transfer coefficient $\lambda \approx \mu \theta$, where $\mu$ is the fiber-fiber friction coefficient. If the critical $\langle\lambda\rangle \sim N^{-1}$, this suggests that for $N \gg 1$ one can interpret the percolation threshold as a lower bound to the total fiber turning angle, specifically $\mu\langle\Theta\rangle \gtrsim 7$ where $\Theta \equiv N \theta$. This quantifies Galileo's assertion about ropes, since twisting fibers together builds $\Theta$, and it parenthetically explains why spinning is such an essential part of the manufacturing process for yarns. As a sanity check for cotton, $\mu \approx 0.3-0.4[4,5]$, and thus, $\langle\Theta\rangle \gtrsim 20$. Table I estimates the total fiber turning angle for fibers in the

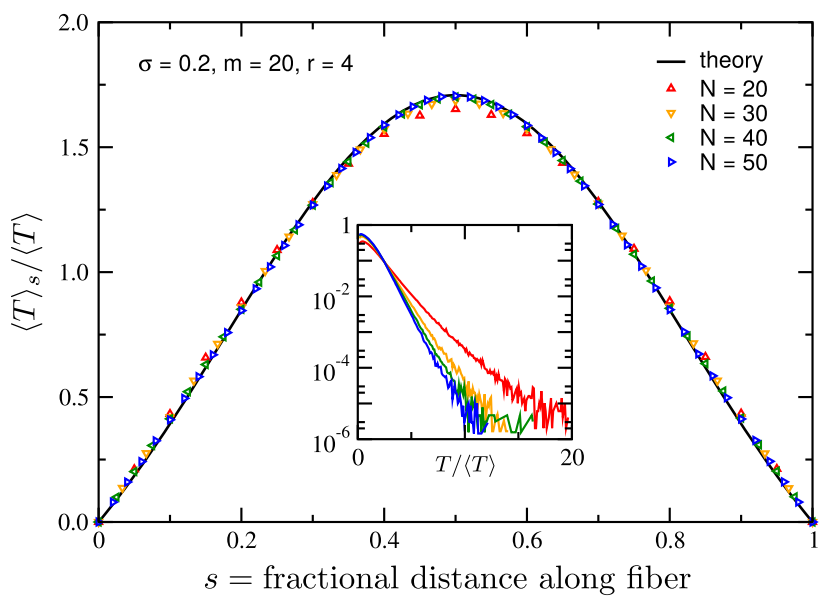

FIG. 4. The mean tension as a function of distance along a fiber, for a baseline parameter set, computed just above the percolation transition. The theoretical curve is the solution to Eq. (2) at $\Lambda=\Lambda_{c}$. The inset shows the distribution of tensions in individual fiber elements (i.e., between pinning points).

yarns in Fig. 1, and it seems that this constraint is indeed comfortably met.

If we interpret tension transmission as a phase transition, it is natural to seek an order parameter. The load will not do, as the problem as specified is homogeneous in the tensions. Instead, for each contact, we use the mean tension $T_{m}$ introduced previously to define the slack, $S=\lambda T_{m}-|\Delta T|$, as the amount by which the tension transferred undershoots the friction limit [18]. The system-wide mean slack $\langle S\rangle$ is an order parameter. In the supercritical state, there is generally not a unique set of tensions (cf. selection of $x$ in the splice toy model), rather there is a feasible solution space, which in this case is an open polytope: a convex, high-dimensional cone in the positive hyper-quadrant of the space of fiber element tensions. To compute $\langle S\rangle$, we select a random edge of the solution cone and average over such edges. The results (Fig. 5) support the notion of a secondorder phase transition in the limit of long fibers [19], although there are significant finite-size effects.

To understand the nature of the percolation transition, we now develop a mean field theory for the tension $T(s)$ in a fiber as a continuous function of fractional arc length $s$. We

TABLE I. The diameter and pitch (10-90 percentile ranges) of 3 -ply Gütermann sewing thread from imaging (cf. Fig. 1 inset). A proxy estimate for the fiber turning angle is $\Theta \approx \kappa l$. The yarn curvature $\kappa$ is estimated by modeling the ply centerline as a helix with diameter $d / 2$ and pitch length $3 \lambda$ [17].

\begin{tabular}{llll}
\hline \hline Diameter & $d$ & $0.23-0.27$ & $\mathrm{~mm}$ \\
Apparent pitch & $\lambda$ & $0.30-0.39$ & $\mathrm{~mm}$ \\
Yarn curvature & $\kappa$ & $1.7-2.5$ & $\mathrm{~mm}^{-1}$ \\
Fiber length [5] & $l$ & $20-30$ & $\mathrm{~mm}$ \\
Fiber turning angle & $\Theta$ & $30-70$ & radians \\
\hline \hline
\end{tabular}




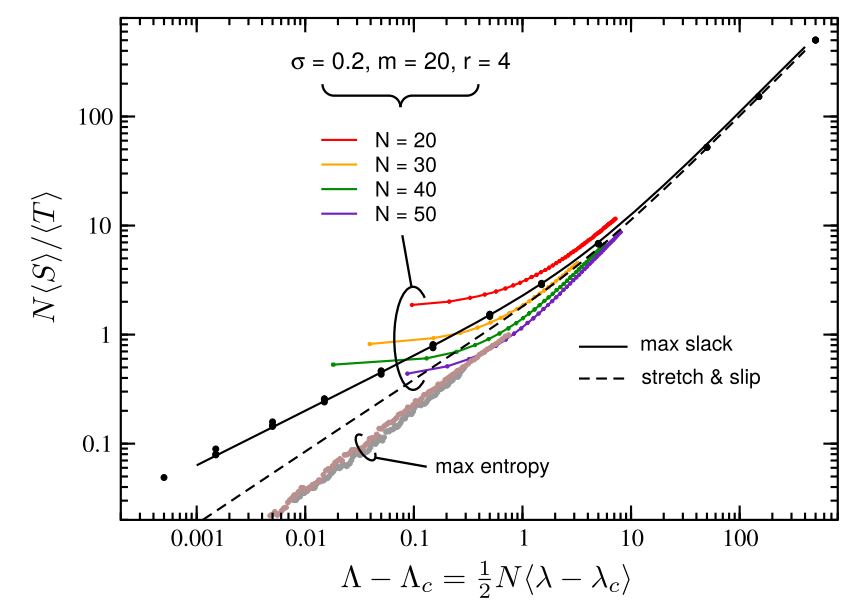

FIG. 5. Normalized slack per fiber versus the departure from criticality. The "max slack" data points are numerical results from three samples each with one self-coupled fiber $N=2000$; the matching theoretical curve is Eq. (3) with no adjustable parameters. The lower dashed line is for the more physical stretch-andslip scenario. The common asymptote at large $\Lambda$ is trivially $N\langle S\rangle=\Lambda\langle T\rangle$. The max-entropy data points show the slack averaged over the microcanonical ensemble of all admissible tensions at fixed $\langle T\rangle=1$, for two samples of a single selfcoupled fiber with $N=2000$. The lassoed rainbow data sets are from the yarn models of Fig. 3, averaged over edges of their solution cone: these are consistent with approaching the maxentropy results at large $N$.

assume $N \gg 1$, and correspondingly, the tension transfer coefficient $\lambda \ll 1$. The centerpiece of the theory is the bidimensional function $\psi\left(s, s^{\prime}\right)$, which gives the actual tension transfer between fibers in contact at $s$ and $s^{\prime}$, i.e., as $\Delta T=\psi \lambda T_{m}$ with $|\psi| \leq 1$. In these terms, $T(s)$ satisfies the integro-differential equation

$$
\frac{d T}{d s}=\Lambda \int_{0}^{1} d s^{\prime}\left[\psi\left(s, s^{\prime}\right) T\left(s^{\prime}\right)-\psi\left(s^{\prime}, s\right) T(s)\right],
$$

where $\Lambda=\frac{1}{2} N \lambda$, noting that on average there are $\frac{1}{2} N$ frictional contacts of each type per fiber. We additionally require $T(0)=T(1)=0$. The mean slack is given by $N\langle S\rangle=\Lambda \int d s \int d s^{\prime}(1-|\psi|) T(s)$, where the integral is over the square domain $\left(s, s^{\prime}\right) \in[0,1] \times[0,1]$.

Load bearing is enhanced by transferring tension to the fiber with longer to go, so for $s<s^{\prime}$, we transfer from $s^{\prime}$ to $s$ and vice versa, and at criticality, we must maximize this opportunity. Thus, as an ansatz, we choose $\psi\left(s, s^{\prime}\right)=$ $\operatorname{sgn}\left(s^{\prime}-s\right) \quad$ (and concomitantly, $\langle S\rangle=0$ ). Equation (2) becomes $d T / d s=\Lambda_{c} \int_{0}^{1} d s^{\prime} \operatorname{sgn}\left(s^{\prime}-s\right)\left[T(s)+T\left(s^{\prime}\right)\right]$. The resulting Sturm-Liouville-like problem can be solved, with a normalized solution $T(s)=2 x_{0}^{2}[1-2 x F(x)]$, where $x=x_{0}(2 s-1), F(x)=\int_{0}^{x} d y \exp \left(y^{2}-x^{2}\right)$ (Dawson's integral [20]), and $x_{0} \approx 0.924$ solves $2 x_{0} F\left(x_{0}\right)=1$. The critical value $\Lambda_{c}=4 x_{0}^{2} \approx 3.416$. For the tension profiles in Fig. 4 , a precise agreement with the numerical results is observed; we speculate that the theory becomes exact in the limit of long fibers. The critical value yields $N \lambda_{c} \approx 6.83$, in good agreement with Fig. 3(b), for long fibers.

Turning now to the supercritical behavior, states with slack are under determined by the friction constraints alone. The challenge is to determine the fractional tension transfer $\psi\left(s, s^{\prime}\right)$ in Eq. (2) within the friction constraint that $|\psi| \leq 1$. The results are dependent on the choice of physics in the supercritical state. Here we sketch the main results [21]. For example, maximizing the slack selects $\psi\left(s, s^{\prime}\right)=0$ in a diagonal band $\left|s^{\prime}-s\right|<w$, while retaining the critical form $\psi\left(s, s^{\prime}\right)=\operatorname{sgn}\left(s^{\prime}-s\right)$ outside this. Treating the band as a perturbation $(w \ll 1)$ recovers the critical Sturm-Liouvillelike problem with $\Lambda_{c}$ replaced by $\Lambda /\left(1+\Lambda w^{2}\right)$. As a result, the latter expression must match $\Lambda_{c}$, leading to $1 / \Lambda+w^{2}=1 / \Lambda_{c}$. For this form of $\psi$ we readily find

$$
N\langle S\rangle=2 w \Lambda\langle T\rangle=2\langle T\rangle \sqrt{\left(\Lambda / \Lambda_{c}\right)\left(\Lambda-\Lambda_{c}\right)} .
$$

To test this, we used linear programing to solve the maxslack condition for a single, long fiber transferring tension to itself. The result (Fig. 5) shows good agreement with the theoretical curve in Eq. (3) over six decades [22].

A more physical model is to presume that as we load the sample, contact points displace affinely where they can within a "core" region $\left|s-\frac{1}{2}\right|<w$, and they otherwise slide under locally critical conditions (a "stretch-and-slip" model [4b]). This means that all contacts associated with the "tails" (i.e., $\left|s-\frac{1}{2}\right| \geq w$ ) are at their sliding condition with $\psi\left(s, s^{\prime}\right)=\operatorname{sgn}\left(s^{\prime}-s\right)$, including their contacts with points $s^{\prime}$ in the core. For points $s$ in the core, we have affine deformation, so we anticipate a uniform strain leading to uniform tension. This turns out to be exactly compatible with Eq. (2), choosing $\psi\left(s, s^{\prime}\right)=0$ when both $s$ and $s^{\prime}$ are in the core, and noting that the contributions to $d T / d s$ in the core from left and right tails cancel each other out. This leads to a second curve shown in Fig. 5.

Yet another possible scenario is to postulate that all supercritical states within the allowed solution cone are equally likely (a "max-entropy" model), akin to the microcanonical ensemble in statistical mechanics, or the Edwards' conjecture in granular packings [23]. A numerical investigation of this case is also shown in Fig. 5.

These possibilities lead to different values for the critical exponent in $\langle S\rangle \sim\left(\Lambda-\Lambda_{c}\right)^{\beta}$, ranging from $\beta=1 / 2$ for the max-slack model, Eq. (3), to $\beta \approx 0.75 \pm 0.05$ for the maxentropy case (fitting to a power law). The near-critical behavior of the stretch-and-slip model is $w \propto\left(\Lambda-\Lambda_{c}\right)^{1 / 3}$ and $\langle S\rangle \propto w^{2}$, leading to the intermediate value $\beta=2 / 3$.

To summarize, we propose a generic percolation transition as the explanation for how staple yarns, woven fabrics, sewn garments, and Galileo's rope transmit tension over arbitrary distances. Our assertion is supported by the appearance of a transition in abstract models, where the friction laws are recast as a linear satisfiability problem. 
This transition appears to be second order, although the critical exponents are dependent on physics beyond simple static friction. The abstract model may be generalized and applied in various ways. For example one can investigate fiber blends, with applications to optimizing the properties of functionalized sewing threads. In another direction, failure could be modeled by iteratively breaking the most highly loaded fiber elements (Fig. 4 inset), cf. elastic fiber bundle models [24]. More generally, the LP approach to Amontons-Coulomb friction problems may have applications in stress transmission in granular media, such as sand piles and grain silos.

We thank Simon Johnson for a critical reading of the manuscript, Andrea Ferrante for helpful comments, and Jane Munro-Brown for the SEM image used in Fig. 1. We acknowledge an anonymous referee for insightful questions regarding what happens near the fiber ends. This work was supported in part by an Established Career Fellowship from the EPSRC (REG).

*patrick.warren@unilever.com

T.C.Ball@warwick.ac.uk

${ }^{\star}$ R.E.Goldstein@damtp.cam.ac.uk

[1] G. Galilei, Discorsi e Dimostrazioni Matematiche Intorno a Due Nuove Scienze (Elzevir, Leiden, 1638); our quote is from the translation by H. Crew and A. de Salvio, Dialogues Concerning Two New Sciences (Macmillan, New York, 1914).

[2] J. W. S. Hearle, P. Grosberg, and S. Backer, Structural Mechanics of Fibers, Yarns, and Fabrics (Wiley-Interscience, New York, 1969), Vol. 1.

[3] N. Pan, J.-H. He, and J. Yu, Text. Res. J. 77, 205 (2007).

[4] N. Pan, Text. Res. J. 62, 749 (1992); 63, 504 (1993).

[5] W. E. Morton and J. W. S. Hearle, Physical Properties of Textile Fibres (Textile Institute, Manchester, 1993).

[6] J. Gao, W. D. Luedtke, D. Gourdon, M. Ruths, J. N. Israelachvili, and U. Landman, J. Phys. Chem. B 108, 3410 (2004).

[7] Tension-dominated means $T_{i} \gg T^{*}$ where $T^{*}$ is defined below; in this regime (yarn load $\gtrsim$ few g wgt), bending stiffness can be safely ignored away from the fiber ends.

[8] The results are insensitive to the choice of which fiber is notionally overwrapped, since this subtlety is erased by randomly shuffling the pinning directions. Also, we expect adjacent frictional contacts are elastically decoupled in the tension-dominated regime, justifying the choice to use independent and identically distributed tension transfer coefficients.

[9] I. M. Stuart, Br. J. Appl. Phys. 12, 559 (1961).

[10] The estimate derives from $T^{*}=\mu N$ where the friction coefficient $\mu=O(1)$ for these purposes. The beam equation then gives the normal force $N \approx B / L^{2}$, where the bending stiffness $B \approx 3-35 \mu \mathrm{N} \mathrm{mm}{ }^{2}$ and the fiber "crimp" length scale (or the distance between frictional contacts) we estimate as $L \gtrsim 100 \mu \mathrm{m}$. The bending stiffness in turn derives from the reported specific flexural rigidity of cotton, $0.53 \mathrm{mN} \mathrm{mm}^{2} / \mathrm{tex}^{2}$ [5], in combination with the reported range of cotton fiber linear densities $0.7-2.3$ denier, where 1 denier $=1 \mathrm{~g} / 9000 \mathrm{~m}=0.11$ tex.

[11] H. Alarcón, T. Salez, C. Poulard, J.-F. Bloch, É. Raphaël, K. Dalnoki-Veress, and F. Restagno, Phys. Rev. Lett. 116, 015502 (2016). Note that the giant amplification of their equivalent $T^{*}$ is peculiar to their geometry of interleaved clamped sheets; we do not expect it to apply to fibers in a staple yarn.

[12] This estimate derives from the reported cotton tensile strength $0.2-0.5 \mathrm{~N} /$ tex [5], multiplied by the above linear fiber density, and is in accord with the yarn tensile strength $\sim 1 \mathrm{~kg}$ wgt.

[13] M. E. Fisher, Rev. Mod. Phys. 70, 653 (1998).

[14] See Supplemental Material at http://link.aps.org/ supplemental/10.1103/PhysRevLett.120.158001 for details concerning calculations setting $T^{*}=0$. To see this, note that the percolation threshold corresponds to the appearance of unbounded LP solution(s). By multiplicative rescaling, we can renormalize $T^{*}$ to zero in the problem, without changing the nature of the transition.

[15] B. F. Bayman, Am. J. Phys. 45, 185 (1977); see also J. Walker, Sci. Am. 249, 120 (1983); J. H. Maddocks and J. B. Keller, SIAM J. Appl. Math. 47, 1185 (1987).

[16] Figure 2(a) is redrawn from the entry on knots in the Encyclopadia Brittanica, 11th edition (Encyclopedia Britannica, New York, 1911).

[17] That is to say, $\kappa=(d / 4) /\left[(d / 4)^{2}+(3 \lambda / 2 \pi)^{2}\right]$.

[18] This definition of slack is closely related to, but not exactly the same as, the LP notion of slack.

[19] For another slant on the problem we also monitored the number of iterations of the LP simplex solver required to decide satisfiability as a measure of computational complexity. We find that this exhibits a peak at the critical point, reminiscent of the behavior of the heat capacity at a conventional second-order phase transition.

[20] M. Abramowitz and I. A. Stegun, Handbook of Mathematical Functions (Dover, New York, 1965).

[21] P. B. Warren, R. C. Ball, and R. E. Goldstein (to be published).

[22] The success in extrapolating the perturbation calculation is explained by the fact that Eq. (3) asymptotes to $N\langle S\rangle /$ $\Lambda\langle T\rangle=2 \Lambda_{c}^{-1 / 2}=1 / x_{0} \approx 1.082$; this is almost the correct large $\Lambda$ behavior, $N\langle S\rangle / \Lambda\langle T\rangle=1$.

[23] S. F. Edwards and R. B.S. Oakeshott, Physica A (Amsterdam) 157, 1080 (1989); S. Martiniani, K. Julian Schrenk, K. Ramola, B. Chakraborty, and D. Frenkel, Nat. Phys. 13, 848 (2017).

[24] S. Pradhan, A. Hansen, and B. K. Chakrabarti, Rev. Mod. Phys. 82, 499 (2010). 